\title{
ETSI SmartBAN in Medical IoT
}

\author{
Matti Hämäläinen ${ }^{*}(1)$, Tuomas Paso ${ }^{(1)}$, Lorenzo Mucchi( ${ }^{(2)}$ \\ (1) Centre for Wireless Communications, University of Oulu, Finland \\ https://www.oulu.fi/cwc/ \\ (2) University of Florence, Italy \\ https://www.dinfo.unifi.it/changelang-eng.html
}

\begin{abstract}
In this paper, we are briefly discussing on the ETSI SmartBAN standard and its utilization in medical Internet of Things (mIoT) applications. The ETSI SmartBAN standard is defining physical and medium access control layers (PHY/MAC), as well as semantic interoperability mechanisms and security for smart wireless body area networks (WBAN). SmartBAN is a European initiative towards wider WBAN utilization. Using the technology providing common language for data representation jointly with energy efficient PHY/MAC implementation is seen as an enabler of future smart mIoT, and IoT networks in general. Since SmartBAN is initially targeted to operate in heterogeneous radio environment, it can also jointly operate with future systems, such as $5 \mathrm{G}$ and $6 \mathrm{G}$.
\end{abstract}

\section{Introduction}

Internet of Things (IoT) is fast spreading system concept in modern home and industrial automation sectors. Adopting easy-to-connect, low power consumption devices, which could provide seamless $24 / 7$ monitoring, control and actuation functions for various use-cases could bring wireless sensor nodes almost everywhere. Typically, IoT devices do not need large communications bandwidth and the use-cases are targeted to transfer small amount of data without strict latency requirements. In some cases, neither is the reliability a key issue. At the moment, the widely spread IoT installations are using one of the various Bluetooth versions [1]; Std. IEEE802.15.4 [2], and/or its higher OSI layer solutions, such as Zigbee; or standards relating to IEEE802.11 family, thus $\mathrm{WiFi}$ [3]. These technologies are available also for wireless body area network (WBAN) use, especially [1]. Medical portable devices, such as monitors, etc. are currently lean on [3]. Industrial automation is using [2] but also the other listed technologies are used.

As general IoT solutions are widely spreading into different utilization fields, also wearables and other health and wellbeing related devices carried by humans, or even implanted inside a human, are being more popular. This is the major application area we are concentrating in this paper.

\section{SmartBAN Justification}

In medical IoT (mIoT) solutions, there are dedicated standards available. The oldest one is the Std. IEEE802.15.6-2012 [4]. In Europe, European Telecommunications Standards Institute (ETSI) started the work towards smart body area network standardization under the Technical Committee (TC) SmartBAN in 2013. TC SmartBAN covers several aspects relating to wireless communications within a WBAN. The main focus areas have been in physical layer (PHY), medium access control layer (MAC), heterogeneity management, semantic interoperability and security. The goal is to have a reliable, low-power standard to boost WBAN technology development and adaptation to global mIoT markets, starting from Europe. Due to the increasing number of wireless devices and heterogeneous technologies operating in the same environment requests also need for efficient interoperability management. The frequency spectrum is already crowded, especially in the license free bands, such as industrial, scientific and medical (ISM) bands, and the number of wireless devices is expected to greatly increase in the coming years, so there will be lots of challenges to provide reliable wireless communications. In mIoT use, the reliability and security/secrecy requirements are even more highlighted.

The wider vision in SmartBAN development work is to get it accepted also in other utilization areas than WBAN, such as automotive vehicles, smart homes and offices, and so on. mIoT should not be the only application area the standardized technology will be used. In wearable domain, the rapid technology development makes it possible today to provide services, which twenty years ago required dedicated desktop computer. This allows, for example, easy collection of human vital signs using seamless technology, which is always connected to backbone systems. If needed, the connection can be done even realtime and local data processing is also possible.

Thus, based on the real consumer expectations, there is a real need for devices, which are more robust, consume less energy and are user-friendly. On the other hand, it is preferable to let people live in their own homes instead of being hospitalized. This requires seamless, robust and energy efficient solutions to enable remote control of human health parameters, allowing longer home-staying, e.g., for elderly or outpatients. Not only on-body 
communications, SmartBAN is also studying in-body communications, e.g., for capsule endoscopy purposes.

The technical requirements set to SmartBAN system in general level include the following: SmartBAN devices need to be energy efficient. They need to co-exist with the other radio systems sharing the same area and frequency band. The SmartBAN system needs to fulfil pre-defined quality of service (QoS) requirements, which are given in the SmartBAN system description document. In mIoT applications, the QoS requirements cannot be highlighted too much. Finally, the SmartBAN is providing fast access mechanism for nodes to connect to desired hub. [5]

\section{SmartBAN Architecture}

The current SmartBAN system model is based on a onehop star network topology having up to 16 connected nodes. The maximum range is limited to $2 \mathrm{~m}$. The SmartBAN network is controlled by a network coordinator, called as Hub, and it is the most powerful device associated to the network. The SmartBAN sensor nodes connected to the Hub can consist of reduced functionalities and thus they could consume much less energy and be cheaper.

To connect SmartBAN to other wireless networks, the Hub will have additional functionalities, which are needed, e.g., for routing and other interactions with the co-locating systems. Thus, SmartBAN coordinator should be capable to communicate with heterogeneous networks, being e.g., Bluetooth Low Energy, or cellular such as 3/4/5G or even 6G. A SmartBAN coordinator could be a mobile phone or dedicated device, which can provide enough power and multi-radio service if needed. In the agenda, SmartBAN Technical Committee (TC) has plans also for simpler nodes/gadgets, such as wristbands, smart watches, etc., which could act as a relay or a bridge node, and create connections, e.g., to hidden nodes [5]. However, the SmartBAN coordinator and relaying functionalities are not included yet in the standard, but the work is ongoing.

\section{SmartBAN Physical Layer}

The SmartBAN physical layer is defined in the technical specification [6]. This chapter summaries the key features of the PHY.

SmartBAN is operating at $2.4 \mathrm{GHz}$ ISM band. The use of that frequency band does not need license and is globally available, which makes it easier to take SmartBAN technology in use. On the other hand, $2.4 \mathrm{GHz}$ ISM band is very crowded, which causes some challenges, e.g., to interference and coexistence management.

SmartBAN consists of 40 physical channels having bandwidth of $2 \mathrm{MHz}$, allocating the operation frequency from $2401 \mathrm{MHz}$ to $2481 \mathrm{MHz}$ with center frequencies $f_{\mathrm{c}}$ [6]

$$
f_{\mathrm{c}}=2402 \mathrm{MHz}+2 * n \mathrm{MHz}, n=0 \ldots 39 \text {, }
$$

where $n$ is the channel number. Three channels, Ch0, Ch12 and $\mathrm{Ch} 39$, are dedicated to control information and they are named as Control Channels $(\mathrm{CCH})$. The other 37 channels are reserved for data payload, and the channels are called as Data Channels (DCH).

Hub is using $\mathrm{CCH}$ to broadcast control channel beacon $(\mathrm{C}$ Beacon), which introduces the following information describing the network: Hub address, slot length, time slots, interference mitigation mechanism used, duty cycling, DCH channel number, initial state and time stamp. In addition, the $\mathrm{CCH}$ Beacon frame has one reserved slot for future amendments. All the data, as well as control and management information, is transmitted using bidirectional $\mathrm{DCH}$. One established SmartBAN is using only one $\mathrm{CCH}$ and $\mathrm{DCH}$. The data channel beacon (D-Beacon) is periodically sent by Hub using the data channel allocated for the user for data transmission. Interference mitigation and coexistence managements with adjacent SmartBAN networks are handled using different DCHs per each network. The current version of SmartBAN deviates, e.g., from Bluetooth as it is not supporting frequency hopping. [7]

As reliable communications is one of the key enablers of SmartBAN, the standard has defined forward error control coding and repetitions for improving the transmission quality. $\mathrm{BCH}(36,22,2)$ shall be employed to protect Physical Layer Convergence Protocol (PLCP) Header and the MAC Protocol Data Unit (MPDU) may be protected using $\operatorname{BCH}(127,113,2)$. In the case, where more error correction capability is required, repetition coding may be utilized for the whole Physical-Layer Protocol Data Unit (PPDU) by repeating PPDU block either twice or four times. E.g., in [4], repetition coding is done bit-wise. The SmartBAN PPDU packet format is shown in Figure 1 [7].

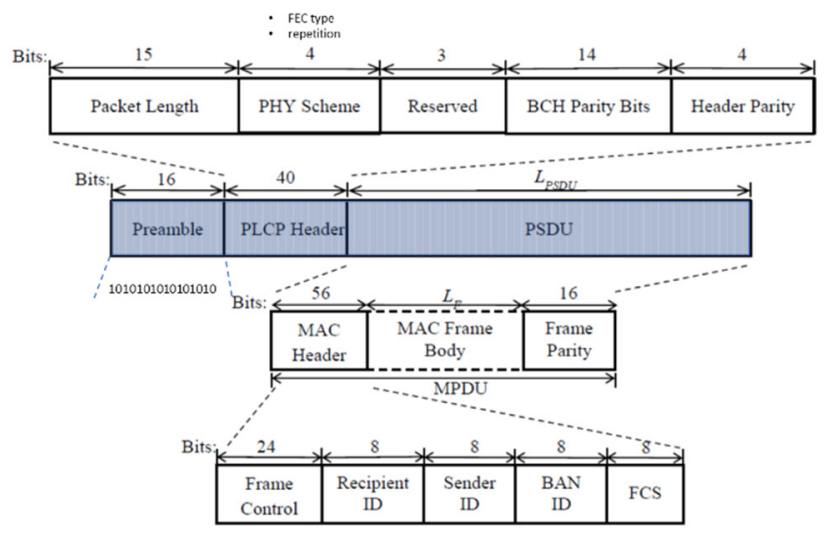

Figure 1. SmartBAN PPDU format.

For data modulation, SmartBAN is utilizing Gaussian frequency shift keying (GFSK). In GFSK, the modulation index $h=0.5$ and bandwidth-bit period product $B T=0.5$ are used [6]. Data rate is nominally $1 \mathrm{kbps}$ to $100 \mathrm{kbps}$ for vital sign monitoring, but it is scalable up to $1 \mathrm{Mbps}$ 
without $\mathrm{BCH}$ coding for the MPDU and without repetition coding [5]. The standard does not give any transmission power limitation, but it should follow the one defined for ISM-band license-free devices. In addition, specific absorption ratio (SAR) limits for the devices operating in the close vicinity of a human body need to be followed.

\section{SmartBAN Medium Access Control Layer}

One of the goals in SmartBAN work was to define a simple MAC, which still enables efficient functionalities, especially for mIoT applications. The main block in MAC layer is a superframe termed as Inter-Beacon Interval (IBI). The superframe is divided into three parts: scheduled access period (SAP), control and management period $(\mathrm{C} / \mathrm{M})$ and inactive period, as shown in Figure 2, where $T_{\mathrm{s}}$ and $T_{\mathrm{D}}$ are depicting slot time and length of the IBI period, respectively. The number of available slots in SAP and $\mathrm{C} / \mathrm{M}$ are marked as $N_{\mathrm{S}}$ and $N_{\mathrm{CM}}$, respectively. Time division multiple access (TDMA) based SAP is reserved for data transmission and contention free channel access is provided for different users. Control and management messaging is carried out in the $\mathrm{C} / \mathrm{M}$ period using slotted Aloha. The SmartBAN superframe allows also using C/M period for data transmission if there are not enough resources available at SAP. During the inactive period, transmissions are not allowed as it is introduced to decrease power consumption, since devices can sleep during it. To support prioritized communications, SmartBAN introduces four user priority levels; low, mid, high and very high (emergency) priority. The standard defines different contention probabilities for different user priorities. [7]

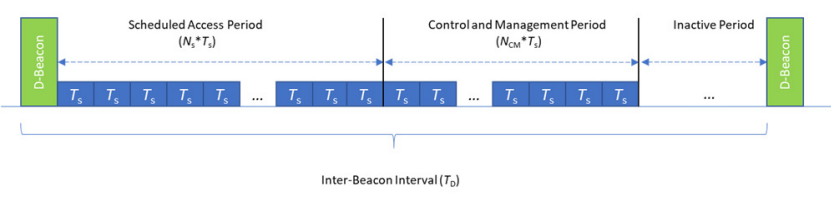

Figure 2. SmartBAN IBI structure in DCH.

As an option, SmartBAN introduces a novel channel access method called as Multi-use Channel Access (MCA). MCA provides very low-latency emergency messaging for time critical applications using Priority Channel Access (PCA). In addition, MCA enables the utilization of the scheduled but unused time slots during the SAP thus increasing the channel capacity and decreasing the latency. This feature is called as Re-use Channel Access (RCA). As MCA is not mandatory channel access mechanism, its implementation is left for vendors. However, if any node in the SmartBAN is using MCA, all the other nodes connected to the same network needs to use it. On the other hand, the standard defines that MCA functionalities need to be supported by all Hubs. [8]

Both features, the PCA and the RCA, are enabled by the unique time slot structure defined for the MCA, as shown in Figure 3. The MCA slot structure introduces sensing period for Clear Channel Assessment (CCA) at the beginning of each slot. The CCA is performed for two different purposes. When MCA is enabled, all nodes have to perform a CCA before transmitting a packet in their allocated slot during the SAP, or before sending a control or management frame during the $\mathrm{C} / \mathrm{M}$ period, to detect a possible emergency transmission, which always starts at the beginning of the slot. Furthermore, nodes having an outstanding packet in their queue can access the channel during every slot of the SAP, i.e. before their allocated slot, if they perform a CCA to detect if the slot owner has started a transmission or not. In the latter case, two CCAs is thus performed. CCA can be based on threshold detection, carrier sensing or both. After channel sensing, a back-off mechanism is defined for busy channel cases.

As shown in Figure 3, Inter-frame spaces (IFS) are used to distinguish data frames from acknowledgement frames (ACK), as well as consecutive slots. [6]

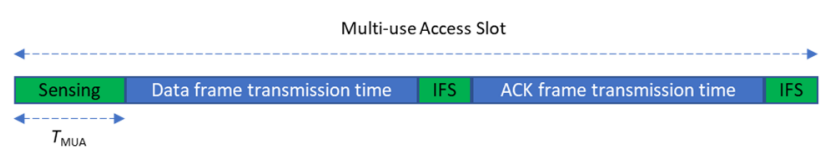

Figure 3. MCA slot structure.

\section{SmartBAN Interoperability}

The current multivendor mIoT device supply produces various user interfaces, data descriptions and representations as well as profiles. This means that it is not guaranteed that data produced by different manufacturers' devices are analogous.

For interoperability and heterogeneity management, SmartBAN has defined mechanisms in [9]. The SmartBAN is targeting for data-level, semantic and technical interoperability. This approach enables efficient protocol interfaces in various levels and cross-domain use-cases. The proposed interoperability takes into account also security aspects as the most motivating use-cases come from the health and wellbeing sectors. SmartBAN defines open data reference semantic model, which can be associated with metadata. The functionalities are supporting both measurements and control. Due to the harmonized data presentation, SmartBAN provides also semantic analysis, which enables automated monitoring and controlling. As the standard defines unified representations for data and corresponding metadata, which are aligned with Bluetooth Low Energy medical devices' profile, SmartBAN could easily coexist and share data between these multi-technology wireless medical devices. [9]

In 5G, healthcare and wellness are included in the listed key application verticals. It is expected that the demands, performance and usage of wireless healthcare technologies are increasing in the near future. If seeing beyond $5 \mathrm{G}$, just started 6G programme will increase and merge the utilization of heterogeneous technologies in various application areas. E.g., in [10], extended use of biosensors 
and wearables is predicted, jointly with seamless graphical user interfaces, distributed calculations, and so on.

From the medical data origin, at the close vicinity of human, SmartBAN will offer a technology to serve also the future needs. Interoperability issues are taken into account in the SmartBAN work from the beginning enabling SmartBAN to have an important role in the future health and wellness related applications. As future 5G/6G technologies are increasing the possible ways to connect WBANs to wider communication networks, the SmartBAN coordinator can act as a bridge between the human centric measurements and a wider, ubiquitous medical access network.

\section{SmartBAN use-cases}

ETSI TC SmartBAN has defined various use-cases where the technology is targeted to be used [5]. The listed usecases are dealing with person's safety monitoring, when vital signs can be automatically collected and transmitted to electrical health records from where the data can be accessed by the authorized health professionals. Corresponding uses-cases are such as stress, sleep and fall monitoring. For patients having, e.g., cardiac diseases, automated cardiac rhythm or blood pressure monitoring would be an important feature. As WBANs support mobility, wearables and thus, SmartBAN, can be used in various sports and welfare applications to collect human's vital information during the training periods. In assisted living environments, SmartBAN is a suitable technology for smart living and smart home solutions to provide additional information of the person's wellbeing and lifestyle. Due to the various needs for quality-of-service different applications have, the SmartBAN system is scalable in the means of data rates, latency requirements, allowed errors, and so on.

Beyond-5G and, in particular 6G technology, will enable the on-body and in-body networks as part of Internet. Nanoscale dry (artificial) and wet (biological) devices operating inside or on the surface of the human body will be part of the connectivity fabric, which $6 \mathrm{G}$ systems aims to include. This opportunity opens new outstanding services, especially in the health sector. SmartBAN envisions this kind of future BANs. The coordinator will act as a smart interface between the body world and the Internet world. The radio technology outwards from SmartBAN hub can be selected from the existing and coming standards, which best fits to the service requirements and provides needed quality. Thus, SmartBAN is also future-proof WBAN technology. The listed issues were example use-cases and challenges SmartBAN is going to tackle.

\section{Conclusion}

In this paper, the general characteristics of ETSI SmartBAN, the European standard for smart body area networks is given. The Technical Committee has published standards for physical and medium access control layers, semantic interoperability and open data models. SmartBAN is targeted to health and wellness related applications, which needs very reliable, low latency communications jointly with very low power consumption.

As initially targeted to support interoperability between various technologies, SmartBAN is eligible to operate also with new systems, such as $5 \mathrm{G}$ and $6 \mathrm{G}$. Both new technologies include healthcare as one of their major application verticals, which means that there will be even higher need for energy efficient technologies at WBAN side. SmartBAN will be the first element in the whole transmission chain, which produces data for other technologies.

More information about ETSI TC SmartBAN can be found from https://www.etsi.org/technologies/smart-body-areanetworks.

\section{Acknowledgements}

This research has been financially supported in part by the University of Oulu's self-funded project WBAN Communications in the Congested Environments (MeCCE), by Academy of Finland 6Genesis Flagship (grant 318927) and by the European Union's Horizon 2020 programme under the Marie Skłodowska-Curie grant agreement No. 872752. We would also like to give big thanks to the whole ETSI TC SmartBAN colleagues involved in the process.

\section{References}

[1] https://www.bluetooth.com/

[2] IEEE Standards Association, "IEEE Standard for Low-Rate Wireless Personal Area Networks (WPANs). IEEE Std 802.15.4 "TM-2015."

[3] IEEE Standards Association, "Part 11: Wireless LAN Medium Access Control (MAC) and Physical Layer (PHY) Specifications. IEEE Std 802.11 TM-2016."

[4] IEEE Standards Association, "Part 15.6: Wireless Body Area Networks. IEEE Std 802.15.6-2012."

[5] Smart Body Area Networks (SmartBAN); System Description. ETSI TR 103394.

[6] Smart Body Area Network (SmartBAN); Enhanced Ultra-Low Power Physical Layer. ETSI TS 103326.

[7] Smart Body Area Network (SmartBAN); Low Complexity Medium Access Control (MAC) for SmartBAN. ETSI TS 103325.

[8] J. Haapola, T. Paso, and R. Kohno, "Method of Improving Channel Utilization", US Patent 9565692, 07.02.2017.

[9] Smart Body Area Networks (SmartBAN); Unified data representation formats, semantic and open data model. ETSI TS 103378.

[10] M. Latva-aho, K. Leppänen (eds.), "Key Drivers and Research Challenges for 6G Ubiquitous Wireless Intelligence - 6G Research Visions 1", September 2019. 